\title{
DYNAMIC PARAMETERS OF ELASTIC PLATE OPTICAL SWITCH DRIVES
}

\author{
Vagif Ali Magerramov, \\ Baku Technical University, Baku,Azerbaijan,mvg476@mail.ru \\ Mehman Huseyn Hasanov, \\ Baku Technical University, Baku, Azerbaijan, mhasanovnew@gmail.com
}

DOI: $10.36724 / 2664-066 X-2020-6-2-20-23$

\begin{abstract}
The methods and means of improving the efficiency and the parameters of the dynamic parameters of the elastic plate of the optical commutator drive using advanced information and telecommunication technologies are analyzed. The bandwidth of optical communication networks based on systems with flat spring-loaded optical drive switch rods is investigated. On the basis of the study, the dynamic parameters of the elastic plate of the optical commutator drive are proposed a structural-functional scheme of a system with flat spring-loaded plates and linear algebraic equations for the dynamics of an elastic plate with the aid of which the equation for small oscillations of a rod near a rectilinear position is compiled. The equations of dynamics of systems with flat spring-loaded optical drive switch rods are considered and determined. On the basis of the system-technical analysis, a general integral of the spring deflection equation is determined. With the help of the solution, the equation for the dynamics of an elastic plate obtained a mathematical expression of the displacement of the upper end of the rod vertically of the spring-loaded drive system of the optical commutator.
\end{abstract}

KEYWORDS: dynamics equations, optical commutator drive, integral of the deflection equation, fiber-optic networks

Information about authors

Vagif Ali Magerramov, Doctor of Mathematics, Professor, The department "Radiotexnika and televisons systems", Baku Technical University, Baku, Azerbaijan

Mehman Huseyn Hasanov, Cand. Tech Science, Associate Professor, The Department "Multi-channel telecommunication systems", Baku Technical University, Baku, Azerbaijan 


\section{Introduction}

The main advantage of fiber-optic networks is their practically unlimited bandwidth. The practical value of this property lies in the possibility of a multiple increase in the speed of information transmission over fiber-optic communication channels on a global scale. This makes research in the field of optical networks very relevant and promising.

Systems with flat spring-loaded (Fig. 1) plates and other microelectromechanical and piezoceramic drives [1,2] can be used as a drive in the switch.

As noted in [1], each linear-translational motion drives under the action of signals from the switch control unit can be in a passive (recessed) or active (vertical) position.

As can be seen from Figure 1, a semitransparent mirror in the passive position can pass the optical beam (Fig. 1a), or deflect the beam at an angle of 900 , dividing it into two equal flows (Fig. 1b).

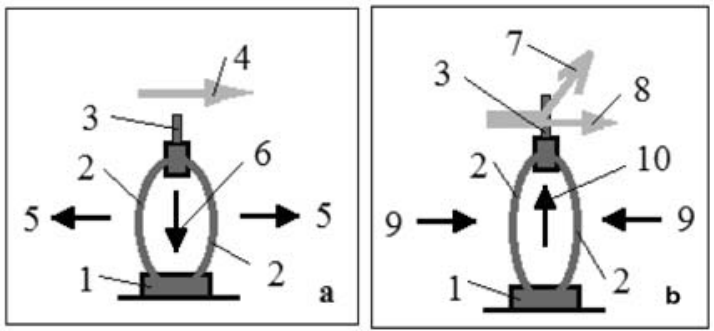

Figure 1. Kinematics of a system with flat spring loaded plates:

1 - base; 2 - spring-loaded plates; 3 -translucent mirror; 4 -optical beam of direct passage; 5 - pulling force of the plates; 6 -direction of decreasing the height of the system (drowning the translucent mirror) downward; 7 -deflected beam; 8 - beam passed through a semitransparent mirror; 9 -force of compression of plates; 10 -directions of increasing the height of the system (falling of the translucent mirror) up.

A distinctive feature of the system with flat spring-loaded plates (Fig. 1) is that due to the spring-loaded drive [3], when the acting voltage is removed, the system returns to its original (initial) position. In the system, both electromagnetic and piezoelectric translational drive can be used as a drive.

\section{1. uation of dynamics of an elastic plate}

Consider a system with flat spring-loaded plates [3], in which there are two elastic plates $\mathrm{A}$ and $\mathrm{B}$ with a given length $L$ and rigidity $C$. On them, as shown in Fig. 1a, a semitransparent mirror of mass $m$ is attached. To obtain the dynamic equation of this system $\pm F(t)$, we divide the spring-loaded plates into two parts along the line as shown in Fig. 1b. We will assume that acting on one of the plates with a force, we thereby force it to contract or lengthen (respectively, the other plate will contract or lengthen), and the height of the plate (in Fig.1b) is shortened or lengthened by $\pm \Delta x$. The force exerts a similar effect $\pm F(t)$ on the other plate.

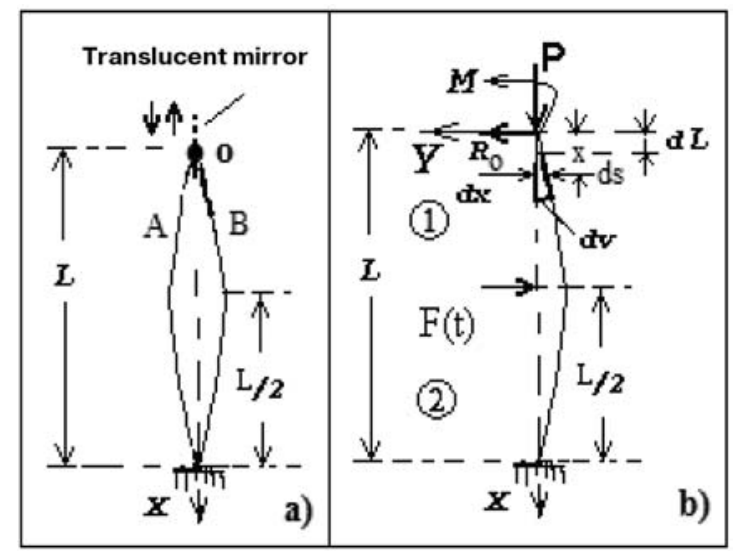

Figure 2. Kinematics of an elastic plate

In the given problem, the elastic rods under the action of the force $\pm F(t)$, the gravity force $m$ of the mirror and the rigid support, as well as the conditions of attachment at the ends, will be in conditions of longitudinal-transverse bending (Fig. 2b). Since the end of the rod is considered rigidly fixed, then when the rod is curved, in addition to the reaction force $R_{0}$, there is also an end moment $M_{0}$. The force $P$ is equal to half the weight of the mirror and the rigid support $P=m g / 2$. The second part of the weight of the mirror and rigid support comes from the other rod.

Compose the equation of small vibrations of the rod near the rectilinear position. We assume that the distributed mass of the rod is negligible in comparison with the mass of the mirror and the rigid support, while neglecting the resistance forces, the equation of small vibrations has the form [4]:

$$
E I \frac{\partial^{2} \vartheta}{\partial x^{2}}=-p \vartheta+M
$$

where $v(x, t)$ - dynamic deflection at each point, $E$ - modulus of elasticity, $I$ - moment of inertia, $M(x, t)$ - dynamic bending moment arising in the cross-sections of the bar as a result of transverse load and support reactions.

We divide the rod into two (1 and 2) sections, then the bending moment will be:

in the first section $M_{1}=R_{0}(t)-M_{0}(t)$

in the second section $M_{2}=R_{0}(t) x-M_{0}(t)+F_{2}(t)\left(x-\frac{L}{2}\right)$

Then, in the first section, we get the following differential equation:

$$
\frac{\partial^{2} \vartheta_{1}}{\partial x^{2}}+K_{0}^{2} \vartheta_{1}=\frac{1}{E I}\left[-R_{0} X-M_{0}\right]
$$

in the second section: 


$$
\frac{\partial^{2} \vartheta_{2}}{\partial x^{2}}+K_{0}^{2} \vartheta_{2}=\frac{1}{E I}\left[-R_{0} X-M_{0}+F\left(X-\frac{L}{2}\right)\right]
$$

where the notation is introduced: $K_{0}^{2}=P / E I ; \quad P=m g / 2$.

It is known that [5], the equations of small oscillations can be satisfied with the expressions:

$$
\begin{aligned}
& \vartheta(x, t)=V(x) e^{i \cdot \omega \cdot t} \\
& F_{2}(t)=F e^{i \cdot \omega \cdot t} \\
& R_{0}(t)=R_{0} e^{i \cdot \omega \cdot t} \\
& M_{0}(t)=M_{0} e^{i \cdot \omega \cdot t}
\end{aligned}
$$

here $\omega$ is some constant.

Substituting expressions (6) into (4) and (5), get: in the first section:

$$
\frac{d^{2} V_{1}}{d x^{2}}+K_{0}^{2} V_{1}=\frac{1}{E I}\left(-R_{0} X-M_{0}\right)
$$

in the second section:

$$
\frac{d^{2} V_{2}}{d x^{2}}+K_{0}^{2} V_{2}=\frac{1}{E I}\left[-R_{0} X-M_{0}+F\left(X-\frac{L}{2}\right)\right]
$$

Then, the general integral of equations (7) and (8), respectively, will be:

$$
\begin{gathered}
V_{1}(x)=C_{1} \operatorname{Sin}_{0} X+C_{2} \operatorname{Cos} K_{0} X-\frac{1}{E I K_{0}^{2}}\left(R_{0} X+M_{0}\right) \\
V_{2}(x)=C_{3} \operatorname{Sin}_{0} X+C_{4} \operatorname{Cos} K_{0} X-\frac{1}{E I K_{0}^{2}}\left[R_{0} X+M_{0}-F\left(X-\frac{L}{2}\right)\right]
\end{gathered}
$$

As can be seen from (9) and (10), in each section the deflection linearly depends on the constant parameters in the first and second sections: $C_{1}, C_{2}, C_{3}, C_{4}, R_{0}$ and $M_{0}$.

These constant coefficients are determined from the conditions for fixing the plate at the ends and the conditions of continuity during the transition from the first section to the second.

In the case considered in Fig. $2 \mathrm{~b}$ case, these conditions are as follows:

$$
\begin{array}{cccc}
\text { at } & x=0 & V_{1}(0)=0 & V_{1}^{I}(0)=0 \\
\text { at } & x=L / 2 & V_{1}(L / 2)=V_{2}(L / 2) & V_{1}^{I}(L / 2)=V_{2}^{I}(L / 2) \\
\text { at } & x=L & V_{2}(L)=0 & V_{2}^{I}(L)=00
\end{array}
$$

Using boundary conditions (11) from (9) and (10) after some transformations [4], we obtain a system of six linear algebraic equations, from the solution of which we finally obtain the desired constant coefficients:

$$
\begin{aligned}
& C_{1}=\frac{F}{2 K_{0} P} ; \\
& C_{2}=-\frac{F}{2 K_{0} P} \frac{\operatorname{Sin}(\alpha / 2)}{\operatorname{Cos}(\alpha / 2)} ; \\
& C_{3}=-\frac{F}{2 K_{0} P} \frac{\operatorname{Cos}(3 \alpha / 2)}{\operatorname{Cos}(\alpha / 2)} ; \\
& C_{4}=\frac{F}{2 K_{0} P} \frac{\operatorname{Sin}(3 \alpha / 2)}{\operatorname{Cos}(\alpha / 2)} ; \\
& R_{0}=\frac{F}{2} ; \\
& M_{0}=-\frac{F}{2 K_{0}} \frac{\operatorname{Sin}(\alpha / 2)}{\operatorname{Cos}(\alpha / 2)}
\end{aligned}
$$

where $\quad \alpha=L \cdot \sqrt{\frac{3 \cdot m \cdot g}{2 \cdot b \cdot h^{3} \cdot E}}-$ constant parameter, $b$ - width and $h$ - plate thickness.

Then the general integral of the deflection equation, in the first and second sections, respectively, will be:

$$
\begin{aligned}
& V_{1}(x)=C_{1}\left(\operatorname{Sin}_{0} X-K_{0} X\right)+C_{2}\left(\operatorname{Cos}_{0} X-1\right) \\
& V_{2}(x)=C_{3} \operatorname{Sin}_{0} X+C_{4} \operatorname{Cos}_{0} X-C_{1} K_{0} X-C_{2}+\frac{F}{P}\left(X-\frac{L}{2}\right)
\end{aligned}
$$

Taking into account (12) in (13), finally, in the first section get:

$$
V_{1}(x)=\frac{F \cdot L}{4 P} \cdot A_{1}(x)
$$

where

$$
A_{1}(x)=\frac{1}{\alpha}\left[\operatorname{Sin} K_{0} X+\operatorname{tg} \frac{\alpha}{2}\left(1-\cos K_{0} X\right)-K_{0} X\right]
$$

Taking into account (12) in (14), finally, in the second section get:

$$
V_{2}(x)=\frac{F \cdot L}{4 P} A_{2}(x)
$$

where

$$
A_{2}(x)=\frac{1}{\alpha}\left[\frac{\operatorname{Sin}\left(\frac{3 \alpha}{2}-K_{0} X\right)}{\operatorname{Cos} \frac{\alpha}{2}}+K_{0} X-2 \alpha+\operatorname{tg} \frac{\alpha}{2}\right]
$$




\section{V tical offset of the upper end of the bar}

One of the important parameters of the optical commutator drive with the proposed flat spring-loaded rods is the displacement of the upper end of the rod on which the semitransparent mirror is fixed (position 3 in Fig. 1). Therefore, after determining the deflection, we determine the vertical displacement of the upper end of the bar.

With lateral deflection, the centerline of the bar is initially compressed, and the upper part of the spring-loaded system is somewhat lengthened. We assume that the rod is not extensible. Therefore, consider an element $d S$ of the curved axis of the beam (Fig. 2b). Let $d X$ is its projection onto the axis $X$, then there will be a bend $d V$. The vertical offset of the top end of the bar will be:

$\Delta L=\int_{0}^{L} d L=\int_{0}^{L}(d S-d X)=\int_{0}^{L}\left[\left(\sqrt{1+\left(\frac{d V}{d X}\right)^{2}}\right) d X-d X\right] \approx \frac{1}{2} \int_{0}^{L}\left(\frac{d V}{d X}\right)^{2} d X ;$

To determine the vertical displacement of the upper end of the bar $\Delta L$, we use solutions (9) and (10). Then the total rod elongation has:

$$
\Delta_{\mathrm{L}}=\Delta_{\mathrm{L} 1}+\Delta_{\mathrm{L} 2}
$$

where

$$
\begin{aligned}
& \Delta L_{1}=\frac{1}{2} \int_{0}^{L}\left(\frac{d V_{1}}{d X}\right)^{2} d X \\
& \Delta L_{2}=\frac{1}{2} \int_{L / 2}^{L}\left(\frac{d V_{2}}{d X}\right)^{2} d X
\end{aligned}
$$

Taking into account (15) in (19) and (16) in (20) and after transformation, we finally obtain the mathematical expression for the vertical displacement of the upper end of the rod of the spring-loaded optical commutator drive system:

$$
\Delta L=\frac{F^{2}}{16 p^{2}} \cdot L \cdot D(\alpha) e^{2 \cdot i \cdot \omega \cdot t}
$$

where

$D(\alpha)=\operatorname{tg}^{2}\left(\frac{\alpha}{2}\right)+3+\frac{1}{\alpha}\left[(2 \cos \alpha+1) \operatorname{tg} \frac{\alpha}{2}-2 \sin \alpha\right]$

Next, an expression can be obtained for the displacement of the upper end of the rod of the spring loaded drive system or the upper end of the semitransparent mirror of the optical switch.

For this case, we will have [5]:

$$
\begin{gathered}
\Delta L_{1}=\frac{F(t)^{2} L}{32 P^{2}}\left[\operatorname{tg}^{2} \frac{\alpha}{2}+3+\frac{4}{\alpha}(\cos \alpha+1) \operatorname{tg} \frac{\alpha}{2}-\frac{4}{\alpha} \sin \alpha\right] e^{2 i \omega t} \\
\Delta L_{2}=\frac{F(t)^{2} L}{32 P^{2}}\left[\operatorname{tg}^{2} \frac{\alpha}{2}+3-\frac{2}{\alpha} \operatorname{tg} \frac{\alpha}{2}\right] e^{2 i \omega t}
\end{gathered}
$$

Taking into account (22) and (23) in (18), the expression describing the displacement of the semitransparent mirror is obtained as

$$
\Delta L=\frac{F(t)^{2} \cdot L}{16 P^{2}} \cdot D(\alpha) \cdot e^{2 i \omega t}
$$

Considering that $F(t)=F \cdot e^{i \omega t}$, find:

$$
F(t)=4 \cdot P \sqrt{\frac{\Delta L}{L \cdot D(\alpha)}}=2 m g \sqrt{\frac{\Delta L}{L \cdot D(\alpha)}}
$$

where $\Delta L$ - displacement of the top end of a rod or translucent mirror.

Similarly, by imposing such particular solutions, one can obtain a formula for the external action $F(t)$ for the general case of transverse vibrations.

To determine the greatest transverse deflection of the bar $V_{\text {max }}$, we will use the condition for fixing the plate. As noted, the function $V(x)$ reaches its maximum value when $X=L / 2$, then:

$$
V_{1}\left(\frac{L}{2}\right)=V_{2}\left(\frac{L}{2}\right)=V_{M A X}=\frac{F(t)}{4 P} \cdot L \cdot D_{1}(\alpha)
$$

where

$$
D_{1}(\alpha)=\frac{2}{\alpha} \cdot \operatorname{tg}\left(\frac{\alpha}{2}\right)-1
$$

Excluding the value of the external action from relation (26), we represent the largest transverse deflection in a different form:

$$
V_{M A X}=D_{1}(\alpha) \cdot \sqrt{\frac{L \cdot \Delta L}{D(\alpha)}}
$$

Also, it can be shown that:

$$
\begin{aligned}
& \Delta L=\frac{D(\alpha)}{D_{1}^{2}(\alpha)} \cdot \frac{V_{M A X}^{2}}{L}=\frac{F(t)}{4 p} \cdot \frac{D(\alpha)}{D_{1}(\alpha)} \cdot V_{M A X} \\
& F(t)=4 p \cdot \frac{V_{M A X}}{L \cdot D_{1}(\alpha)}=4 p \frac{D_{1}(\alpha)}{D(\alpha)} \frac{\Delta L}{V_{M A X}}
\end{aligned}
$$

Thus, all mathematical expressions for the dynamic parameters (such as the deflection equations $V(L)$, external force $F(t)$ and displacement of the upper end $\Delta L$ ) of the spring-loaded system from the parameters of the elastic plate ( $L-$ length, $b-$ width, $h$ - thickness and $m$ - masses of the elastic plate with a semitransparent mirror) of the optical commutator drive.

\section{References}

1. Maharramov VA, Gasanov M.G. About one principle of commutation of information flows. Problems of Information Technology, Baku, 2018. No.1, pp. 27-35.

2. Hasanov M.H. Multi-channepiezoelectric switch of adaptiveoptical networks. The International science-technical journal Herald of the Azerbaijan Enginering Academy. Baku, 2017. №4. pp. 107-112.

3. Ismailov T.K., Magerramov V.A. Scanning device, Auto. Svid. No. 1283698 A2 of the USSR, B.I., No.2. MKI G02 B26 / 10. 1987.

4. Timoshenko S. P. Oscillation in engineering. Moscow: Fizmatgiz. 1967. 444p.

5. Maharramov V. A. Technique of infrared observations of space objects (Fundamentals of theory and calculation). Baku. Elm. 1999. 336 p. 